\title{
Vaccine-associated measles in a healthy 40-year-old woman
}

\author{
Lucas Churchill BSc, Francesco A. Rizzuti MD, Kevin Fonseca PhD, Joseph Kim MD
}

Cite as: CMAJ 2018 September 4;190:E1046-8. doi: 10.1503/cmaj.180527

A 40-year-old woman, originally from Armenia, presented to the emergency department with a three-day history of fever, malaise, myalgia and nonproductive cough, followed by a rash that began on her face and spread downwards to her trunk and arms. She had delivered her first child nine weeks earlier with no complications. Ten days before onset of symptoms, she had received both mumps-measles-rubella (MMR) (MMR II, Merck Frosst Canada) and tetanus-diphtheria vaccinations. The patient lacked childhood immunization records and reported that she had not received all of her childhood vaccinations in Armenia owing to an allergic reaction, which remained unrecognized on her immigration to Canada.

Apart from prior subacute thyroiditis, the patient was healthy and was taking no active medications. She had no recent travel or known sick contacts. She had immigrated to Canada seven years prior, was married to a monogamous male partner and worked as an accountant.

On initial examination, she was alert and oriented. She was normotensive, but tachycardic at 116 beats $/ \mathrm{min}$. She was febrile with a maximum temperature of $39.3^{\circ} \mathrm{C}$. A mild conjunctival injection was noted. She had a diffuse blanchable erythematous maculopapular rash involving her face, trunk and proximal upper extremities (Figure 1). The rest of her examination was noncontributory. Laboratory investigations showed mild leukopenia (leukocytes 2.6 [normal 4.0-11.0] $\times 10^{9} / \mathrm{L}$ ) and elevated lactate dehydrogenase (320 [normal 100-235] U/L). Chest radiography was unremarkable.

An acute respiratory viral infection, along with the possibility of measles, was considered based on the fever and rash presentation. A nasopharyngeal swab and urine sample were sent for respiratory viruses and measles virus testing. On admission, the patient was placed in an airborne isolation room, along with additional precautions.

On postadmission day one, the respiratory viral panel came back negative for the following: influenza $A$ and $B$; respiratory syncytial virus; parainfluenza virus 1, 2, 3 and 4; adenovirus; coronaviruses 229E, NL63, OC43 and HKU1; and the enterovirus/ rhinovirus group. Both the nasopharyngeal and urine samples tested positive for measles virus by real-time polymerase chain reaction ( $\mathrm{PCR}$ ), which targets the hemagglutinin and nucleoprotein genes of the virus. ${ }^{1}$ Differentiation between the wild-type

\section{KEY POINTS}

- Vaccination with the measles-mumps-rubella vaccine is safe, but can very rarely be associated with clinically significant illness that is indistinguishable from wild-type measles.

- Rapid molecular testing to differentiate between wild-type and vaccine-associated measles is of paramount importance in guiding decisions about infection prevention and control, and public health at the local level.

- The risk of transmission of vaccine-associated measles is low, and it may not pose a risk to susceptible newborns who are exposed during the infectious period of the illness.
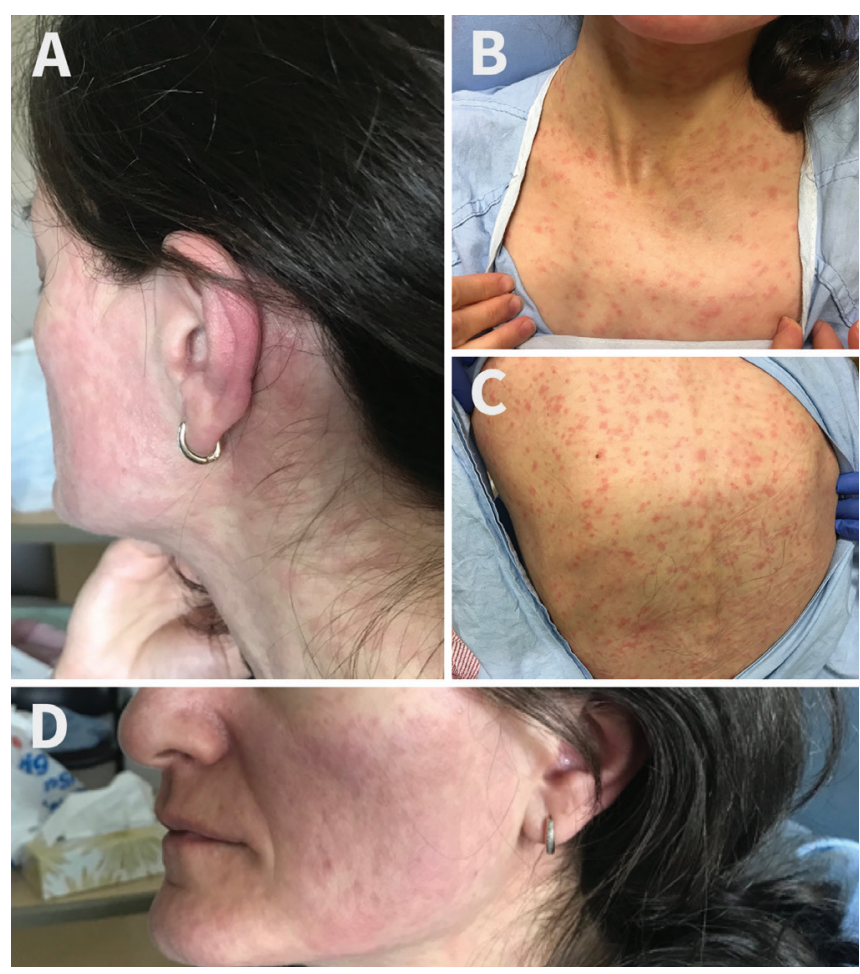

Figure 1: Initial presentation of a 40-year-old woman to the emergency department three days after onset of symptoms, with diffuse, blanchable, nonpruritic maculopapular rash on the face and postauricular area $(A)$, neck and chest (B) and back (C). On postadmission day two (five days after symptom onset) the rash was resolving (D). 
and vaccine strains was performed by an in-house assay. The results were available postadmission day two and showed that it was a vaccine strain of measles virus. Subsequently, the sample was sent to the National Microbiology Laboratory of Canada, which established that the virus belonged to genotype A, confirming it to be a vaccine strain.

By postadmission day two, the patient showed clinical improvement with resolution of fever. After consultation with public health, and infection prevention and control staff, no exposure follow-up was done and additional precautions were discontinued. At the time of discharge, the patient's rash was beginning to fade. A telephone interview at three months postdischarge showed that the patient and her infant were doing well. The infant had been followed by her own physician in the interim and no concerns were noted.

\section{Discussion}

Measles is a highly contagious viral infection, typically of childhood, associated with substantial morbidity and mortality worldwide. The introduction of a two-dose schedule of MMR vaccine in 1996 has been critical in interrupting endemic transmission of measles in Canada. There are, however, sporadic cases of measles still occurring, mostly in undervaccinated populations. ${ }^{2}$ Whereas local reactions at the injection site can be common, about $5 \%$ of children vaccinated with MMR will experience fever and malaise (with or without rash). ${ }^{3}$ Adverse events are thought to be more common with the first dose of MMR vaccine. Despite these recognized adverse effects, the benefits of the MMR vaccine far outweigh the risks. Vaccination with the MMR vaccine is contraindicated in immunocompromised patients, in whom serious complications such as pneumonitis and measles inclusionbody encephalitis have been observed. ${ }^{4}$ The MMR vaccine also should not be given during pregnancy.

Our patient had her first MMR vaccine as an adult. The decision to offer MMR vaccine in this case was consistent with the current recommendation that susceptible mothers be offered vaccination postpartum. ${ }^{3}$ Unfortunately, our patient developed a major adverse event necessitating a hospital admission. Her clinical presentation was indistinguishable from wild-type measles. Retesting of her previous prenatal serology showed that she had no evidence of serologic protection against measles, suggesting that this was unlikely to be modified measles (i.e., attenuated infection in patients with pre-existing but limited measles immunity). Only one confirmed case of vaccine-associated measles with classic presentation of fever and morbilliform rash in an immunocompetent adult has been reported in the literature. This case, documented by Kurata and colleagues, involved a 23-year-old healthy man who developed late-onset vaccine-associated measles 20 days postvaccination. ${ }^{5}$

Discriminating between cases of wild-type and vaccineassociated measles is critical in guiding the public health, and infection prevention and control response. Given its high communicability, wild-type measles requires extensive contact investigation and follow-up, whereas vaccine-associated measles would be considered less infectious and would not necessitate subsequent intervention. ${ }^{6}$ Although at that time there were no reports of measles in our health region or an obvious epidemiologic link to another measles case, we could not rule out wild-type measles in our patient. The decision to initiate a public health, and infection prevention and control response hinged on determining the strain of measles virus. This was a time-sensitive matter given the short window for identifying susceptible contacts and administering immunoprophylaxis. Such an investigation would have had substantial implications including resource use, and the indirect cost of forced absenteeism from school, work or any other public activity, as well as the risk associated with immunoprophylaxis. These issues are aptly illustrated in a case report by Hau and colleagues, wherein the local public health department was required to respond to a case of vaccine-associated measles as if it were wild-type measles because of delays in genotyping confirmation. ${ }^{7} \mathrm{Had}$ molecular testing been made more readily available in this case, the lengthy and costly process of contact tracing and follow-up could have been averted.

Once measles virus is detected, the only definitive way to discriminate between wild-type measles and vaccine-associated measles is through molecular testing. ${ }^{8}$ Our institution had ready access to in-house PCR and genotyping, which confirmed the infection to be a vaccine strain within 24 hours. This assay targets a small nucleotide polymorphism in the hemagglutinin gene and detects a small nucleotide polymorphism that is unique to the Schwarz and Moraten vaccine strains, used in North America and much of Europe. ${ }^{9}$ Without the availability of this local assay, the confirmation from the national reference laboratory would have taken several days. This rapid result allowed us to avoid an intensive and resource-consuming public health, and infection prevention and control response.

In circumstances where viral genotyping is not readily available, we suggest that the decision to initiate a contact investigation should be made with careful consideration of epidemiologic risk factors (e.g., the likelihood of wild-type measles circulating in the local community) and clinical context (e.g., recent MMR vaccination history). If the probability of vaccine-associated measles is high, a multidisciplinary decision might limit the scope of investigation until confirmatory testing is done.

Although the live-attenuated vaccines are generally thought to be noninfectious, there is a theoretical risk of viral shedding and potential transmission, especially among immunocompromised household contacts who may be susceptible. ${ }^{3}$ There have been case reports suggesting transmission of yellow fever vaccine virus, another live-attenuated vaccine, from mothers to infants through breast milk. ${ }^{10}$

Our patient was not breastfeeding at the time of presentation. However, the communicability of vaccine-associated measles was an issue of particular concern in our case since our patient maintained intimate contact with her two-month-old daughter, who would not yet have been vaccinated with MMR, during the potentially infectious period of her illness. Infants, having yet to develop a fully competent immune system, represent perhaps one of the most vulnerable populations susceptible to measles infection. ${ }^{3,8}$ Nevertheless, a recent systematic review in 2016 found no evidence to suggest that live-attenuated measles vaccine virus is transmissible. ${ }^{6}$ 
Based on this review of the literature and previous cases of vaccineassociated measles in our health region, it was decided that immunoprophylaxis would not be provided to the infant.

Our patient's child did not develop a febrile illness resembling measles. This outcome corroborates the assumption that vaccine-associated measles is noninfectious and poses negligible risk to contacts, regardless of their immune status or age. Our case supports the safety of MMR vaccination among household contacts of a vulnerable population and the precedent that contact investigation and postexposure prophylaxis are not necessary in confirmed cases of vaccine-associated measles.

\section{References}

1. Hummel KB, Lowe L, Bellini WJ, et al. Development of quantitative genespecific real-time RT-PCR assays for the detection of measles virus in clinical specimens. J Virol Methods 2006;132:166-73.

2. Sherrard L, Hiebert J, Cunliffe J, et al. Measles surveillance in Canada: 2015. Can Commun Dis Rep 2015;42:139-45. Available: www.canada.ca/en/public-health/ services/reports-publications/canada-communicable-disease-report-ccdr/ monthly-issue/2016-42/ccdr-volume-42-7-july-7-2016/ccdr-volume-42-7-july-7 -2016-we-stop-measles.html (accessed 2017 Dec. 4).

3. Page 12: Canadian Immunization Guide: Part 4 - Active vaccines. Ottawa: Public Health Agency of Canada; (modified 2007 Oct. 20). Available: www.canada.ca/ en/public-health/services/publications/healthy-living/canadian-immunization -guide-part-4-active-vaccines/page-12-measles-vaccine.html (accessed 2017 Dec. 4).

4. Bitnun A, Shannon P, Durward A, et al. Measles inclusion-body encephalitis caused by the vaccine strain of measles virus. Clin Infect Dis 1999;29:855-61.

5. Kurata T, Kanbayashi D, Kinoshita $\mathrm{H}$, et al. Late onset of vaccine-associated measles in an adult with severe clinical symptoms: a case report. Am J Med 2014;127:e3-4.
6. Greenwood KP, Hafiz R, Ware RS, et al. A systematic review of human-tohuman transmission of measles vaccine virus. Vaccine 2016;34:2531-6.

7. Hau M, Schwartz KL, Frenette $C$, et al. Local public health response to vaccineassociated measles: case report. BMC Public Health 2013;13:269.

8. Public health disease management guidelines: measles. Edmonton: Government of Alberta; 2013 (modified 2018 May 29). Available: https://open.alberta. ca/publications/measles (accessed 2017 Dec. 4).

9. Bankamp B, Takeda M, Zhang Y, et al. Genetic characterization of measles vaccine strains. J Infect Dis 2011;204(Suppl 1):S533-48.

10. Kuhn S, Twele-Montecinos L, MacDonald J, et al. Case report: probable transmission of vaccine strain of yellow fever virus to an infant via breast milk. CMAJ 2011;183:E243-5.

Competing interests: None declared.

This article has been peer reviewed.

The authors have obtained patient consent.

Affiliations: Departments of Medicine (Churchill, Rizzuti, Kim), and Microbiology, Immunology and Infectious Diseases (Fonseca), University of Calgary; Provincial Laboratory for Public Health (Fonseca), Alberta Health Services, Calgary, Alta.

Contributors: All of the authors contributed to writing and revising the article, gave approval of the final manuscript and agreed to act as guarantors of the work.

Acknowledgements: The authors thank Dr. Raymond Tellier from the Provincial Laboratory for Public Health and Dr. Judy MacDonald, Medical Officer of Health, Alberta Health Services, for their assistance with the case and manuscript preparation.

Correspondence to: Joseph Kim, joskim@ucalgary.ca 